\title{
THE SYMBOLICAL THEORY OF FINITE EXPANSIONS*
}

O. E. GLENN

\section{INTRODUCTION.}

The first two sections of this paper are the result of an attempt to identify, and state in general terms, certain fundamental common properties of a large number of known types of finite expansions of polynomials in $p$ variables. The number of such expansions which are known and available for such a synthesis is of course large, and only the most salient properties can be comprised under one general theory. The principles proved in these preliminary sections are subjected to verification when it is shown that they hold for a number of new expansions. These are derived in sections 3 to 6 inclusive. They are of considerable interest in themselves, apart from the general theory, to the fundamental laws of which they conform.

The Aronhold symbolical notation $\dagger$ for algebraic forms subject to linear transformations constitutes the basis of the general methods of the paper.

To state briefly our initial point of view; if a function $f$ can be given an expression of the form

$$
f=B_{0} A_{1}^{m}+m B_{1} A_{1}^{m-1} A_{2}+\left(\begin{array}{c}
m \\
2
\end{array}\right) B_{2} A_{1}^{m-2} A_{2}^{2}+\cdots+B_{m} A_{2}^{m},
$$

it can be represented symbolically as an $m$ th power, say

$$
f=\Xi_{\Lambda}^{m}=\left(\Xi_{1} A_{1}+\Xi_{2} A_{2}\right)^{m}=\Xi_{1}^{m} A_{1}^{m}+m \Xi_{1}^{m-1} \Xi_{2} A_{1}^{m-1} A_{2}+\cdots .
$$

But with $\nabla_{1}=\Xi_{2}\left(\partial / \partial \Xi_{1}\right)$ this may be written

$$
f=\Xi_{1}^{m} A_{1}^{m}+\frac{\nabla_{1} \Xi_{1}^{m}}{L} A_{1}^{m-1} A_{2}+\frac{\nabla_{1}^{2} \Xi_{1}^{m}}{\underline{L}} A_{1}^{m-2} A_{2}^{2}+\cdots+\frac{\nabla_{1}^{m} \Xi_{1}^{m}}{\underline{m}} A_{2}^{m} .
$$

Now the symbol $\mathbf{m}_{1}^{m}$ equals $B_{0}$. If it prove possible to find a non-symbolical operator $\nabla$ equivalent $(\$ 1)$ to $\nabla_{1}$, then we shall have expressed $f$ in the funda.

- Presented to the Society, April, 1911, and March, 1913.

† Aronhold, Journal far Mathematik, vol. 55 (1858), p. 97. 
mental, non-symbolical form

$$
f=B_{0} A_{1}^{m}+\frac{\nabla B_{0}}{\underline{1}} A_{1}^{m-1} A_{2}+\frac{\nabla^{2} B_{0}}{\underline{2}} A_{1}^{m-2} A_{2}^{2}+\cdots+\frac{\nabla^{m} B_{0}}{\underline{m}} A_{2}^{m} .
$$

This is what is actually done in what follows.

\section{§ 1. Fundamental Lemma.}

Definitions. The general term function will be used to indicate an algebraic form in $p$ variables.

By an expansion of a function we shall mean a representation of it as a finite series proceeding by a law in powers of one or more arguments, with coefficients dependent upon the coefficients, and possibly upon the variables also (\$3), of the original function.

Equivalent operators. In connection with the familiar symbolical representation of a binary form

$$
\begin{aligned}
f=a_{x}^{n}=\left(\alpha_{1} x_{1}+\alpha_{2} x_{2}\right)^{n} & =\alpha_{1}^{n} x_{1}^{n}+n \alpha_{1}^{n-1} \alpha_{2} x_{1}^{n-1} x_{2}+\cdots \\
& =a_{0} x_{1}^{n}+n a_{1} x_{1}^{n-1} x_{2}+\cdots,
\end{aligned}
$$

we note that the expressions $\alpha_{1}^{n}\left(=a_{0}\right), \alpha_{1}^{n-1} \alpha_{2}\left(=a_{1}\right), \cdots$ and linear combinations of these, are the only expressions in the symbolical $\alpha_{i}$, which are defined in terms of the actual coefficients. We call an expression, as $I=\alpha_{1}^{n}$ $+p \alpha_{1}^{n-2} \alpha_{2}^{2}$, the symbolical equivalent of the corresponding expression in the $a^{\prime}$ s, $J=a_{0}+p a_{2}$.

The differential operator $\alpha_{2}\left(\partial / \partial \alpha_{1}\right)$ is such that when

$$
\alpha_{2} \frac{\partial}{\partial \alpha_{1}} I=K
$$

and $I$ is a defined expression, then $K$ is a defined expression. Let the nonsymbolical equivalent of $K$ be $L$, that of $I$ being $J$ as illustrated above. Then the non-symbolical operator

$$
O=n a_{1} \frac{\partial}{\partial a_{0}}+(n-1) a_{2} \frac{\partial}{\partial a_{1}}+\cdots+a_{n} \frac{\partial}{\partial a_{n-1}}
$$

has the property that*

* For example when $I$ is the particu'ar expression given above, equations (i), (ii) are respectively

$$
\begin{aligned}
\alpha_{2} \frac{\partial}{\partial \alpha_{1}}\left(\alpha_{1}^{n}+p \alpha_{1}^{n-2} \alpha_{1}^{n}\right) & =n \alpha_{1}^{n-1} \alpha_{2}+p(n-2) \alpha_{1}^{n-3} \alpha_{2}^{3}, \\
O\left(a_{0}+p a_{2}\right) & =n a_{1}+p(n-2) a_{3} .
\end{aligned}
$$

The operands on the left are equivalent, and the right-hand members are also equivalent expressions. 


$$
O J=L .
$$

Two operators, one symbolical and the other non-symbolical, related like $\alpha_{2}\left(\partial / \partial \alpha_{1}\right)$ and $O$ will be called equivalent or isomorphic operators. We shall refer to this important property of equivalence of two operators as the property $(A)$, and we now state it in such general terms as will completely define it for all the symbolical representations of functions that are employed in the sequel.

$(A)$ Let there be given a definite symbolical representation of a function $f$. A symbolical derivative operator on an expression in the defined combinations of the symbols, which produces a result containing the symbols in defined combinations only, has corresponding to it a non-symbolical derivative operator which carries the non-symbolic equivalent of the first expression into that of the second.

Finite Expansions. Any finite expansion of a function $f$ can be made the basis of a symbolical representation of that function. For, if there exists an expansion of $f$ in powers of a given argument $A$,

$$
f=B_{0}+\left(\begin{array}{c}
m \\
1
\end{array}\right) B_{1} A+\left(\begin{array}{c}
m \\
2
\end{array}\right) B_{2} A^{2}+\cdots+B_{m} A^{m},
$$

then $f$ may be represented as the $m$ th power of a purely symbolical binomial expression

$$
\boldsymbol{\Xi}_{1}+\boldsymbol{\Xi}_{2} A \text {. }
$$

That is, if we write $\Xi_{(\Delta)}$ as an abbreviation of this non-homogeneous (as to $A$ ) expression, then

(1') $f=\Xi_{(\Lambda)}^{m}=\left(\Xi_{1}+\Xi_{2} A\right)^{m}=\Xi_{1}^{m}+m \Xi_{1}^{m-1} \Xi_{2} A+\cdots=B_{0}+m B_{1} A+\cdots$.

Then the only defined expressions in the symbols are

$$
\Xi_{1}^{m-k} \Xi_{2}^{k}\left(=B_{k}\right) \quad(k=0,1, \cdots, m),
$$

and linear combinations of these, where, according to our definition of an expansion, the $B_{k}$ are non-symbolical, determinate expressions in the coefficients and variables of $f$.

Symbolical basis. In the case of every finite expansion with which we shall deal, (i) the property $(A)$ holds for both operators

$$
\nabla_{1}=\Xi_{2} \frac{\partial}{\partial \Xi_{1}}, \quad \nabla_{2}=\Xi_{1} \frac{\partial}{\partial \Xi_{2}},
$$

and (ii) the non-symbolical equivalents of $\nabla_{i}$ can actually be found, expressed, not in terms of the unknown $B_{k}$, but in terms of the coefficients of the known function $f$, and the known argument $A$. 
When (i), (ii) have actually been verified we say that the existence of a symbolical basis of the expansion, viz.,

has been verified.

$$
\Xi_{(A)}=\Xi_{1}+\Xi_{2} A \text {, }
$$

Fundamental lemma. For an expansion for which a symbolical basis exists we now prove a lemma. Evidently

Hence ( $\left.1^{\prime}\right)$ gives

$$
\nabla_{1} \Xi_{1}^{m-k} \Xi_{2}^{k}=(m-k) \Xi_{1}^{m-k-1} \Xi_{2}^{k+1} \quad(k=0,1, \cdots, m) .
$$

$$
f=\Xi_{1}^{m}+\nabla_{1} \Xi_{1}^{m} A+\frac{\nabla_{1}^{2} \Xi_{1}^{m}}{L 2} A^{2}+\cdots .
$$

Write $\nabla$ for the non-symbolical equivalent of $\nabla_{1}$, according to the property $(A)$, and set $\Phi=B_{0}\left(=\Xi_{1}^{m}\right)$. Then from ( $\left.2^{\prime}\right)$ we have

Lemma. There exists a function $\Phi$ and a differential operator $\nabla$, capable of being represented symbolically by the linear operator

$$
\nabla_{1}=\Xi_{2} \frac{\partial}{\partial \Xi_{1}},
$$

such that the explicit expansion of $f$ is

$$
f=\Phi+\frac{\nabla \Phi}{L} A+\frac{\nabla^{2} \Phi}{L 2} A^{2}+\cdots+\frac{\nabla^{m} \Phi}{\underline{m}} A^{m} .
$$

If a symbolical basis be assumed in homogeneous form, or, what amounts to the same thing, if $f$ be assumed to have a homogeneous expansion in two arguments $A_{1}, A_{2}$ for which a symbolical basis exists in the form

then

$$
\Xi_{\Delta}=\Xi_{1} A_{1}+\Xi_{2} A_{2},
$$

(3') $f=\Xi_{A}^{m}=\Xi_{1}^{m} A_{1}^{m}+m \Xi_{1}^{m-1} \Xi_{2} A_{1}^{m-1} A_{2}+\cdots=\Phi_{1} A_{1}^{m}+\cdots+\Phi_{2} A_{2}^{m}$.

This is the same as (2) when $A_{1}=1$. But, in general, we have

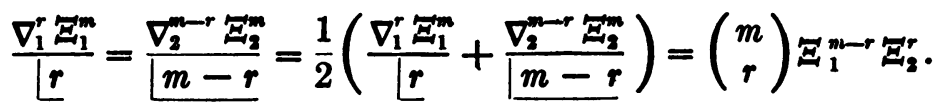

Thus the third expression can be used in place of the general coefficient in $\left(3^{\prime}\right)$. Let $\nabla_{i}^{\prime}$ be the non-symbolical equivalent of $\nabla_{i}(i=1,2)$. Then we get from $\left(3^{\prime}\right)$ an expansion formula more general, and more symmetrical than (2), viz.,

(3) $f=\Phi_{1} A_{1}^{m}+\frac{1}{2}\left(\frac{\nabla_{1}^{\prime} \Phi_{1}}{L 1}+\frac{\nabla_{2}^{\prime m-1} \Phi_{2}}{\underline{m-1}}\right) A_{1}^{m-1} A_{2}+\cdots$

$$
+\frac{1}{2}\left(\frac{\nabla_{1}^{\prime m-1} \Phi_{1}}{\underline{m-1}}+\frac{\nabla_{2}^{\prime} \Phi_{2}}{\underline{1}}\right) A_{1} A_{2}^{m-1}+\Phi_{2} A_{2}^{m} \text {. }
$$


Such an expansion- will be completely given by two non-symbolical operators $\nabla_{i}^{\prime}$ and two pre-determined functions $\Phi_{i}(i=1,2)$.

The importance of a general formula like (3) evidently depends upon the number of expansions which can be found to conform to its laws. We proceed to point out the fact that many of the known finite expansions are special cases of (2) or (3).

(a) Any binary form $f\left(x_{1}, x_{2}\right)=a_{0} x_{1}^{m}+m a_{1} x_{1}^{m-1} x_{2}+\cdots$, when transformed by

$$
x_{1}=\lambda_{1} x_{1}^{\prime}+\mu_{1} x_{2}^{\prime}, \quad x_{2}=\lambda_{2} x_{1}^{\prime}+\mu_{2} x_{2}^{\prime},
$$

gives a result $f_{1}\left(x_{1}^{\prime}, x_{2}^{\prime}\right)$ which is an expansion of $f$ in the arguments $x_{i}^{\prime}$. It is then a case of (3) where now $A_{i}=x_{i}^{\prime}$, and

$$
\begin{aligned}
\Phi_{1}=f\left(\lambda_{1}, \lambda_{2}\right), & \Phi_{2}=f\left(\mu_{1}, \mu_{2}\right), \\
\nabla_{1}^{\prime}=\mu_{1} \frac{\partial}{\partial \lambda_{1}}+\mu_{2} \frac{\partial}{\partial \lambda_{2}}, & \nabla_{2}^{\prime}=\lambda_{1} \frac{\partial}{\partial \mu_{1}}+\lambda_{2} \frac{\partial}{\partial \mu_{2}} .
\end{aligned}
$$

(b) Any covariant of $f\left(x_{1}, x_{2}\right)$, say $C=C_{0} x_{1}^{* 0}+\cdots$, considered as an expansion in $x_{1}, x_{2}$ is a case of (3). Here $\Phi_{1}=C_{0}$, the seminvariant leading coefficient; $\Phi_{2}=C_{w}$. Or $x_{1}^{-w} C$ is a case of (2), where $A=x_{1} / x_{2}$. In either case

$$
\begin{aligned}
& \nabla_{1}^{\prime}=m a_{1} \frac{\partial}{\partial a_{0}}+(m-1) a_{2} \frac{\partial}{\partial a_{1}}+\cdots+a_{m} \frac{\partial}{\partial a_{m-1}} \\
& \nabla_{2}^{\prime}=a_{0} \frac{\partial}{\partial a_{1}}+2 a_{1} \frac{\partial}{\partial a_{2}}+\cdots+m a_{m-1} \frac{\partial}{\partial a_{m}}
\end{aligned}
$$

(c) If $\varphi=\left(a_{0}, a_{1}, \cdots\right)\left(x_{1}, x_{2}\right)^{m}$ goes into $\left(a_{0}^{\prime}, a_{1}^{\prime}, \cdots\right)\left(x_{1}^{\prime}, x_{2}^{\prime}\right)^{m}$ under the special transformation $x_{1}=x_{1}^{\prime}+A x_{2}^{\prime}, x_{2}=x_{2}^{\prime}$, and $I\left(a_{0}, a_{1}, \cdots\right)$ is any rational integral function* of $a_{0}, a_{1}, \cdots$, then the expansion of $I\left(a_{0}^{\prime}, a_{1}^{\prime}, \cdots\right)$ in powers of $A$ is a case of (2), where $f=I\left(a_{0}^{\prime}, \cdots\right), \Phi=I\left(a_{0}, a_{1}, \cdots\right)$, and $\nabla$ is the preceding $\nabla_{2}^{\prime}$.

(d) Taylor's expansion of a non-homogeneous polynomial $f(x)$ in powers of $x-a$ is a case of (2), where

$$
\Phi=f(a), \quad \nabla=\frac{\partial}{\partial a} .
$$

(e) As a proposition inverse to the fundamental lemma (2), or (3), any formal identity containing three terms may be employed as the symbolical basis of finite expansions. In fact, if the identity is

$$
\Xi_{A}=\Xi_{1} A_{1}+\Xi_{2} A_{2},
$$

- Elliott, Algebra of Quantics, p. 115. 
then functions $f=\Xi_{\boldsymbol{A}}^{m}$ and corresponding expansions in the arguments $A_{i}$ exist for all values of $m$. Gordan's* series may be developed (see $\S 6$ ) frcm this point of view, from the formal identity

$$
a_{x} b_{y}-a_{y} b_{x}=(a b)(x y) .
$$

This is also the principle upon which Hermite's theory of typical representation $\dagger$ and associated forms is based.

\section{§ 2. EXTENSION OF THE FUNDAMENTAL LEMMA.}

The extension of (2) to the case of the general homogeneous expansion in $p$ arguments can be readily made. We set

(4') $f=\Xi_{A}^{m}=\left(\Xi_{1} A_{1}+\Xi_{2} A_{2}+\cdots+\Xi_{p} A_{p}\right)^{m}=\Xi_{1}^{m} A_{1}^{m}+\cdots=\Phi_{1} A_{1}^{m}+\cdots$,

and assume that the property $(A)$ is satisfied by all of the symbolical operators of the set

$$
\nabla_{i}=\Xi_{i+1} \frac{\partial}{\partial \Xi_{i}} \quad(i=1,2, \cdots, p-1) .
$$

Then it is easy to verify, by actually performing the indicated differentiations, that $\left(4^{\prime}\right)$ may be written

$$
f=\sum \frac{\nabla_{1}^{i_{1}} \nabla_{2}^{i_{2}} \cdots \nabla_{p-1}^{i_{p-1} \Xi_{1}^{m}}}{\underline{i_{1} \mid}{ }^{i_{2}} \cdots \underline{i_{p-1}}} A_{1}^{m-i_{1}} A_{2}^{i_{1}-i_{2}} \cdots A_{p}^{i_{p-1}},
$$

where the set $\left(m-i_{1}, i_{1}-i_{2}, \cdots, i_{p-2}-i_{p-1}, i_{p-1}\right)$ ranges over the partitions of $m$ into $p$ parts, assuming each partition once and once only. Hence if, according to the property $(A), \nabla_{i}^{\prime}$ is the non-symbolical equivalent of $\nabla_{i}$, then there exists a function $\Phi$ and a set of $p-1$ operators $\nabla_{i}^{\prime}$ such that the explicit expansion of $f$ is $(\S 5)$

$$
f=\sum \frac{\nabla_{1}^{h_{1}} \nabla_{2}^{i_{2}} \cdots \nabla_{p-1}^{\prime i_{p-1} \Phi}}{i_{1}\left|\underline{i_{2}} \cdots\right| i_{p-1}} A_{1}^{m-i_{1}} A_{2}^{i_{1}-i_{1}} \cdots A_{p}^{i_{p-1}} .
$$

\section{3. ON EXPRESSING ONE POLYNOMIAL IN TERMS OF ANOTHER.}

Non-homogeneous variables will be used throughout this section. We let

$$
\begin{array}{r}
f(x)=a_{x}^{m}=a_{0} x^{m}+a_{1} x^{m-1}+\cdots+a_{m} \quad\left(a_{0}=1, m=\mu \nu\right), \\
\varphi(x)=\xi_{x}^{\nu}=\xi_{0} x^{\nu}+\xi_{1} x^{\nu-1}+\cdots+\xi_{\nu} \equiv \xi_{0}\left(x-s_{1}\right)\left(x-s_{2}\right) \cdots\left(x-s_{\nu}\right) .
\end{array}
$$

* Gordan, Vorlesungen uiber Invariantentheorie, vol. 2, \$117.

† Hermite, Journal für Mathematik, vol. 52 (1855), p. 23. 
It may be shown by a form of the Euclidean process of successive division that there exists an expansion of $f$ in powers of $\xi_{x}^{v}$,

$$
f(x)=\Phi_{1}+\Phi_{2} \xi_{x}^{\nu}+\Phi_{3}\left(\xi_{x}^{\nu}\right)^{2}+\cdots+\Phi_{\mu}\left(\xi_{x}^{\nu}\right)^{\mu-1}+\Phi_{\mu+1}\left(\xi_{x}^{\nu}\right)^{\mu},
$$

where $\Phi_{i}$ is a polynomial in $x$ of order $\nu-1$, and in particular, say

$$
\Phi_{1}=\gamma_{0} x^{\nu-1}+\gamma_{1} x^{\nu-2}+\cdots+\gamma_{\nu-1} .
$$

We proceed to the determination of (the coefficients of) $\Phi_{1}$ and the operator $\nabla$ in (2). These will then give the whole expansion, and it will appear that (5) has a symbolical basis of the form

$$
\Xi_{(\phi)}=\Xi_{1 f(x)}+\Xi_{2 j(x)} \varphi .
$$

Substitution of the roots of $\varphi(x)$ for $x$ in (5) gives at once

$$
f\left(s_{i}\right)=\gamma_{0} s_{i}^{\nu-1}+\gamma_{1} s_{i}^{\nu-2}+\cdots+\gamma_{\nu-1} \quad(i=1,2, \cdots, v) .
$$

These are linear equations in the $\gamma_{j}(j=0,1, \cdots, \nu-1)$. Their solution is possible provided $D \neq 0$, where $D$ is the discriminant of $\varphi(x)$. This solution yields

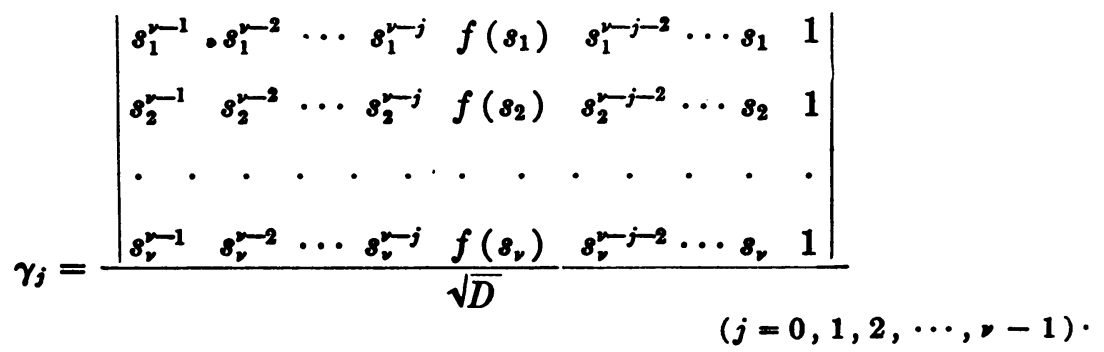

Thus $\gamma_{j}$ is symmetric in the roots $s_{i}$, rational in the coefficients $\xi_{k}(k=0,1, \cdots, \nu)$ and linear in $a_{0}(=1), a_{1}, a_{2}, \cdots, a_{m}$. It is necessary to evaluate the symmetric functions $\gamma_{j}$ of the roots $s_{i}$ in order to determine the operator $\nabla$ required by the theory of the symbolical basis, and (2). I did this originally by means of the Euclidean process mentioned above, i. e., $f(x)$ was divided by $\varphi^{\mu}$, the remainder by $\varphi^{\mu-1}$, and so forth. ${ }^{*}$ The results for the general case are given below, without details of derivation. In these formulas, $\boldsymbol{\Omega}$ indicates the following operator:

$$
\Omega=a_{0} \frac{\partial}{\partial a_{1}}+a_{1} \frac{\partial}{\partial a_{2}}+\cdots+a_{m-1} \frac{\partial}{\partial a_{m}}
$$

- This work was subsequently checked by inductive steps based upon ordinary computation by symmetric functions. 
I obtain

$$
\begin{aligned}
& F=\gamma_{0}=\Omega^{\mu-1} f\left(-\xi_{1}\right)+\sum_{i=2}^{k_{11}} \xi_{i} \Omega^{\mu+i-2} f^{\prime}\left(-\xi_{1}\right) \\
& +\sum_{i=2}^{k_{n}} \sum_{j=2}^{k_{m}} \frac{\xi_{i} \xi_{j}}{2} \Omega^{\mu+i+j-3} f^{\prime \prime}\left(-\xi_{1}\right) \\
& +\sum_{i=2}^{k_{m}} \sum_{j=2}^{k_{n}} \sum_{k=2}^{k_{m}} \frac{\xi_{i} \xi_{j} \xi_{k}}{\mid \underline{3}} \Omega^{\nu+i+j+k-4} f^{\prime \prime \prime}\left(-\xi_{1}\right) \\
& + \text {. } \\
& +\sum_{i=2}^{k_{n}} \sum_{j=2}^{k_{n}} \cdots \sum_{n=2}^{k_{n}} \frac{\xi_{i} \xi_{j} \cdots \xi_{n}}{\lfloor t} \Omega^{n+i+j+\cdots+n-t-1} f^{(t)}\left(-\xi_{1}\right) \\
& \left(k_{r s} \leqq \nu, k_{s 1}+k_{s t}+\cdots+k_{s o} \leqq(\mu-1)_{\nu}+1\right) .
\end{aligned}
$$

Then there results

$$
\begin{aligned}
\Phi=\Phi_{1}= & F x^{\nu-1}+\left[-\left(\xi_{2} \Omega+\xi_{3} \Omega^{2}+\cdots+\xi_{\nu} \Omega^{\nu-1}\right) F+a_{m-\nu+2}\right] x^{\nu-2} \\
& +\left[-\left(\xi_{3} \Omega+\xi_{4} \Omega^{2}+\cdots+\xi_{\nu} \Omega^{\nu-2}\right) F+a_{m-v+s}\right] x^{\nu-s} \\
& +\cdot \cdot \cdot \cdot \cdot \cdot \cdot \cdot \cdot \cdot \cdot \cdot \cdot \cdot \cdot \cdot \cdot \cdot \cdot . \\
& +\left[-\left(\xi_{\nu-1} \Omega+\xi_{\nu} \Omega^{2}\right) F+a_{m-1}\right] x \\
& +\left[-\xi_{\nu} \Omega F+a_{m}\right] .
\end{aligned}
$$

Furthermore, we find during the course of the derivation of these results by the division process that an operator $\nabla$ in (2) exists and is precisely [see (6)]

$$
\nabla=\Xi_{2 f(x)} \frac{\partial}{\partial Z_{1 f(x)}}=-\frac{\partial}{\partial \xi_{v}} .
$$

Hence expansion (5) takes the form

$$
\begin{aligned}
& f(x)=\Phi-\frac{\partial \Phi}{\partial \xi_{\nu}} \xi_{x}^{\nu}+\frac{\partial^{2} \Phi}{\partial\left(\xi_{\nu}\right)^{2}} \frac{\left(\xi_{x}^{\nu}\right)^{2}}{[2}-\cdots+(-1)^{\mu-1} \frac{\partial^{\mu-1} \Phi}{\partial\left(\xi_{\nu}\right)^{\mu-1}} \frac{\left(\xi_{x}^{\nu}\right)^{\mu-1}}{\underline{\mu-1}} \\
& +(-1)^{\mu} \frac{\partial^{\mu} \Phi}{\partial\left(\xi_{\nu}\right)^{\mu}} \frac{\left(\xi_{x}^{\nu}\right)^{\mu}}{\lfloor\underline{\mu}} \text {. }
\end{aligned}
$$

It is thus analogous to the Taylor expansion, to which it reduces when $\nu=1$.

We note in passing the following interesting property of the function $F$ :

$$
-\left(\xi_{1} \Omega+\xi_{2} \Omega^{2}+\cdots+\xi_{v} \Omega^{v}\right) F+a_{m-n+1} \equiv F .
$$




\section{§4. HoMogeneous EXPANSIONS.}

As an illustration of formula (3), we consider the expansion of a homogeneous binary form in terms of two other binary forms of lower order. Let

$$
f_{M}=a_{x}^{M}=\prod_{i=1}^{M}\left(r_{2}^{(i)} x_{1}-r_{1}^{(i)} x_{2}\right)=a_{0} x_{1}^{M}+\cdots
$$

be the form to be expanded. Let the arguments of the expansion be

$$
\begin{aligned}
& A_{1}=f_{1 n}=\alpha_{x}^{n}=\prod_{j=1}^{n}\left(\alpha_{2}^{(j)} x_{1}-\alpha_{1}^{(j)} x_{2}\right)=\alpha_{0} x_{1}^{n}+\cdots, \\
& A_{2}=f_{2 n}=\beta_{x}^{n}=\prod_{j=1}^{n}\left(\beta_{2}^{(j)} x_{1}-\beta_{1}^{(j)} x_{2}\right)=\beta_{0} x_{1}^{n}+\cdots .
\end{aligned}
$$

Then the expansion sought will be of the type

$$
f_{M}=\varphi_{0 p} f_{1 n}^{m}+\varphi_{1 p} f_{1 n}^{m-1} f_{2 n}+\cdots+\varphi_{m p} f_{2 n}^{m},
$$

where $\varphi_{i p}$ is a homogeneous binary form of order $p$.

It is assumed at the outset that $f_{\mathcal{M}}, f_{\text {in }}$ are perfectly general forms, i. e., that their coefficients are independent variables. These forms are also understood to be given in the sense that the coefficient forms $\varphi_{i p}$ are to be determined from them. Hence the totality of coefficients of the $\varphi_{i p}(i=0$, $1, \cdots, m)$ must be equal in number to those of $f_{M}$,

$$
M+1=(m+1)(p+1) \text {. }
$$

Also

Thus

$$
M=m n+p .
$$

$$
p=n-1, \quad M=n(m+1)-1 .
$$

When $M$ and $p$ have these values the solution of $n(m+1)$ linear equations will determine the coefficients of $\varphi_{i n-1}(i=0,1, \cdots, m)$, and consequently the whole expansion. Hence the expansion exists provided these linear equations are consistent. It will be shown that the determinant representing the condition for consistency is a power of the resultant of $f_{1 n}, f_{2 n}$, multiplied by a constant.

For this determinant $D$ is of order $n(m+1)$, and is of the form of the eliminant of the set of $m+1$ forms of order $m n$,

$$
f_{1 n}^{m}, f_{1 n}^{m-1} f_{2 n}, \cdots, f_{2 n}^{m} .
$$

If $D=0$, then there exists a linear relation of the type

$$
\lambda_{1} f_{1 n}^{m}+\lambda_{2} f_{1 n}^{m-1} f_{2 n}+\cdots+\lambda_{m+1} f_{2 n}^{m}=0,
$$


where $\lambda_{m+1}$ is of order $n-1$ in $(x)$, and so $f_{1 n}, f_{2 n}$ have at least one common root. Conversely if $f_{1 n}, f_{2 n}$ have a common root then $D=0$, for otherwise $f_{n(m+1)-1}$ would have a root of multiplicity $m$.

An expansion (13) of $f_{n(m+1)-1}$ exists provided the resultant $R(\alpha, \beta)$ of $f_{1 n}, f_{2 n}$ does not vanish.

To determine our expansion (13), let

$$
\varphi_{i n-1}=p_{i 0} x_{1}^{n-1}+p_{i 1} x_{1}^{n-2} x_{2}+\cdots+p_{i n-1} x_{2}^{n-1}=p_{i x}^{n-1} \quad(i=0,1, \cdots m) .
$$

Substituting $\beta^{(k)}$ for $x$ in (13) we get

$$
p_{0 \beta(k)}^{n-1}\left(\alpha_{\beta(k)}^{n}\right)^{m}=a_{\beta(k)}^{n(m+1,-1} \quad(k=1,2, \cdots n) .
$$

Also owing to the symmetry of $(13)$ in $(\alpha, \beta)$,

$$
p_{m a(k)}^{n-1}\left(\beta_{a(k)}^{n}\right)^{m}=a_{a(k)}^{n(m+1)-1} \quad(k=1,2, \cdots, n) .
$$

Equations (17) form a set of $n$ linear equations for the determination of the $n$ coefficients of $p_{0 x}^{n-1}$. In order to exhibit the solutions as briefly as may be let $\Delta_{\beta}$ be the discriminant of $\beta_{x}^{n}$ and $\Delta_{a}$ that of $\alpha_{x}^{n}$. Then

$$
\pm \Delta_{\beta}=\left|\beta_{1}^{(1) m-1}, \beta_{1}^{(2) m-8} \beta_{2}^{(2)}, \beta_{1}^{(3) m-s} \beta_{2}^{(3))}, \cdots, \beta_{2}^{(n) m-1}\right|^{2} \div \prod_{k=1}^{n} \beta_{2}^{(k) \beta^{(n-1)}}
$$

Also let

$\Sigma_{j}(\alpha, \beta, a)=\mid \alpha_{\beta(1)}^{m n} \beta_{1}^{(1) m-1}, \alpha_{\beta(2)}^{m n} \beta_{1}^{(2) m-2} \beta_{2}^{(2)}, \cdots, \alpha_{\beta())}^{m n} \beta_{1}^{(j) m-1} \beta_{2}^{(j))^{j-1}}$,

then we get

$$
a_{\beta(j+1)}^{n(m+1)-1}, \alpha_{\beta(j+1)}^{m n} \beta_{1}^{(j+2) m-s} \beta_{2}^{(j+2)+2}, \cdots, \alpha_{\beta(n)}^{m n} \beta_{2}^{(n) m-1} \mid \text {, }
$$

$$
p_{0 j}=\frac{1}{R(\alpha, \beta)^{m}} \cdot \frac{\Sigma_{j}(\alpha, \beta, a)}{\prod_{k=1}^{n} \beta_{2}^{(k) m-1} \sqrt{\Delta_{\beta}}} \quad(j=0,1, \cdots, n-1) .
$$

From symmetry, or directly from (18), we have also

$$
p_{m j}=\frac{1}{R(\beta, \alpha)^{m}} \cdot \frac{\sum_{j}(\beta, \alpha, a)}{\prod_{k=1}^{n} \alpha^{(k)-1} \sqrt{\Delta_{\alpha}}} \quad(j=0,1, \cdots, n-1) .
$$

The coefficients of $R(\alpha, \beta)^{-m}$ in (19), (20) are rational, integral and symmetric in the roots of $f_{1 n}, f_{2 n}$ and hence rational in the quantities $\alpha_{0}, \alpha_{1}, \cdots$; $\beta_{0}, \beta_{1}, \cdots$

Referring now to expansion formula (3) we note that the functions $\Phi_{i}(i=1,2)$ are determined in the present case by (19), (20).

The operators $\nabla_{1}^{\prime}, \nabla_{2}^{\prime}$ required by (3) to complete the determination of (13) Tranc. An. Math. 800. 6 
are the Aronhold operators obtained from $\alpha_{x}^{n}, \beta_{x}^{n} ;$ i. e.,

$$
\begin{aligned}
& -\nabla_{1}^{\prime}=\alpha_{0} \frac{\partial}{\partial \beta_{0}}+\alpha_{1} \frac{\partial}{\partial \beta_{1}}+\cdots+\alpha_{n} \frac{\partial}{\partial \beta_{n}}, \\
& -\nabla_{2}^{\prime}=\beta_{0} \frac{\partial}{\partial \alpha_{0}}+\beta_{1} \frac{\partial}{\partial \alpha_{1}}+\cdots+\beta_{n} \frac{\partial}{\partial \alpha_{n}} .
\end{aligned}
$$

These with $\Phi_{1}=\varphi_{0 n-1}, \Phi_{2}=\varphi_{m n-1}, A_{i}=f_{i n}$, give in explicit form the desired expansion of $f_{n(m+1)-1}$; that is, it takes the form (3):

$$
f_{n(m+1)-1}=\Phi_{1} f_{1 n}^{m}+\frac{1}{2}\left(\frac{\nabla_{1}^{\prime} \Phi_{1}}{\lfloor}+\frac{\nabla_{2}^{\prime m-1} \Phi_{2}}{m-1}\right) f_{1-}^{m-1} f_{2 n}+\cdots+\Phi_{2} f_{2 n}^{m}
$$

It is of some interest to enumerate the conditions, necessary and sufficient, in order that series (22), taken in non-homogeneous form with $x_{2}=1$, say, should have constant coefficients. We get a minimum set of such conditions by equating to zero all of the coefficients of $\varphi_{i n-1}(i=0,1, \cdots, m)$ except the last.

Let (19) be written in the form

$$
p_{0 j}=\frac{Q_{j}(\alpha, \beta)}{R(\alpha, \beta)^{m}} \quad(j=0,1, \cdots, n-1) .
$$

Then the aforesaid conditions are given by

$$
C_{i j} \equiv \nabla_{1}^{\prime \prime} Q_{j}=0 \quad\left(\begin{array}{l}
i=0,1, \cdots, m \\
j=0,1, \cdots, n-2
\end{array}\right) .
$$

Their number is

$$
N=(n-1)(m+1) \text {. }
$$

It follows that a series free from conditions $(N=0)$, having constant coefficients, exists only when the arguments $f_{\text {in }}$ are linear $(n=1)$.

By constructing equations analogous to (14) it is easy to show that a ternary form $f_{m}$ can be expressed as a ternary expansion in three ternary arguments $f_{\text {in }}(i=1,2,3)$,

$$
f_{m}=\varphi_{m 00} f_{1 n}^{m}+\cdots,
$$

with the coefficient forms $\varphi$ all of order $n-1$, and with the expansion free from conditions, only when the arguments are linear.

\section{EXPansions with uINEAR aRGUMENTS.}

Consider next the problem of expanding a $p$-ary form $a_{x}^{m}$ in terms of powers of $p$ linear $p$-ary forms $\alpha_{i x}(i=1,2, \cdots, p)$. Expansion formula (4) applies, and

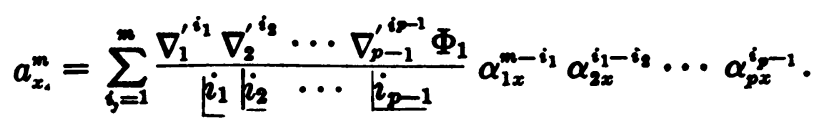


In this the coefficient $\Phi_{j}$ is given by the set

$$
i_{1}=i_{2}=\cdots=i_{j-1}=m ; \quad i_{j}=i_{j+1}=\cdots=i_{p-1}=0 \quad(j=1,2, \cdots, p) .
$$

To get $\Phi_{1}$ we substitute for $(x)$ in (24) the coördinates of the point of intersection of the $p-1$ hyperplanes

This gives

$$
\alpha_{i x}=\alpha_{i 1} x_{1}+\alpha_{i 2} x_{2}+\cdots+\alpha_{i p} x_{p}=0 \quad(i=2,3, \cdots, p)
$$

$$
x_{j}=-\frac{\left(\alpha_{2} \alpha_{8} \cdots \alpha_{j-1} \alpha_{p} \alpha_{j+1} \cdots \alpha_{p-1}\right) x_{p}}{\left(\alpha_{2} \alpha_{8} \cdots \alpha_{p}\right)} \quad(j=1,2, \cdots, p),
$$

and by substitution

$$
\Phi_{1}=\frac{\left(a \alpha_{2} \alpha_{3} \cdots \alpha_{p}\right)^{m}}{\left(\alpha_{1} \alpha_{2} \cdots \alpha_{p}\right)^{m}}
$$

For this expansion there exists a symbolical basis in the form of $\left(4^{\prime}\right)$, as may readily be verified by considering (24) to be the transformed of $a_{x}^{m}$ by the inverse of

$$
X_{i}=\alpha_{i x} \quad(i=1,2, \cdots, p) .
$$

The operators for this case. are the Aronhold operators

$$
-\nabla_{i}^{\prime}=\alpha_{i 1} \frac{\partial}{\partial \alpha_{i+11}}+\alpha_{i 2} \frac{\partial}{\partial \alpha_{i+12}}+\cdots+\alpha_{i p} \frac{\partial}{\partial \alpha_{i+1 p}} \quad(i=1,2, \cdots, p-1),
$$

the whole expansion (24) being given by $\Phi_{1}$ and $\nabla_{i}^{\prime}$.

It can be shown that corresponding results hold for forms whose coefficients belong to a field or reduced residue system* [modd $p, P(x)$ ], where $p$ is a prime number and $P(x)$ an irreducible quantic modulo $p$ of order $n$. Thus when $m=p=2$, we have, provided $(\alpha \beta) \neq 0[\operatorname{modd} p, P(x)]$, an expansion of the form

$$
\begin{aligned}
f \equiv \Phi_{1}\left(\alpha_{1} x_{1}-\alpha_{2} x_{2}\right)^{2}+I\left(\alpha_{1} x_{1}-\alpha_{2} x_{2}\right)\left(\beta_{1} x_{1}-\beta_{2} x_{2}\right) \\
+\Phi_{2}\left(\beta_{1} x_{1}-\beta_{2} x_{2}\right)^{2}[\operatorname{modd} p, P(x)],
\end{aligned}
$$

where the linear arguments have coefficients belonging to the residue system. Then

$$
\begin{aligned}
& \Phi_{1} \equiv\left(\alpha_{1} \beta_{2}-\alpha_{2} \beta_{1}\right)^{p^{n-3}}\left(a_{0} \beta_{2}^{2}+a_{1} \beta_{2} \beta_{1}+a_{2} \beta_{1}^{2}\right), \\
& \Phi_{2} \equiv\left(\alpha_{1} \beta_{2}-\alpha_{2} \beta_{1}\right)^{p-3}\left(a_{0} \alpha_{2}^{2}+a_{1} \alpha_{2} \alpha_{1}+a_{2} \alpha_{1}^{2}\right)
\end{aligned}
$$

$[\operatorname{modd} p, P(x)]$,

* Dickson, Linear Groups, p. 7. 
and with

we have

$$
\nabla_{1}^{\prime}=-\left(\alpha_{1} \frac{\partial}{\partial \beta_{1}}+\alpha_{2} \frac{\partial}{\partial \beta_{2}}\right), \quad \nabla_{2}^{\prime}=-\left(\beta_{1} \frac{\partial}{\partial \alpha_{1}}+\beta_{2} \frac{\partial}{\partial \dot{\alpha}_{2}}\right),
$$

$$
\begin{aligned}
I=\frac{1}{2}\left(\Delta_{1}^{\prime} \Phi_{1}+\nabla_{2}^{\prime} \Phi_{2}\right) \equiv-(\alpha \beta)^{p^{n-3}} & {\left[2 a_{0} \alpha_{2} \beta_{2}+a_{1}\left(\alpha_{1} \beta_{2}+\alpha_{2} \beta_{1}\right)\right.} \\
& \left.+2 a_{2} \alpha_{1} \beta_{1}\right] \quad[\operatorname{modd} p, P(x)] .
\end{aligned}
$$

The form $f=a_{x} b_{x}$ is apolar to $\varphi=\left(\alpha_{1} x_{1}-\alpha_{2} x_{2}\right)\left(\beta_{1} x_{1}-\beta_{2} x_{2}\right)=\alpha_{x} \beta_{x}$ provided $I \equiv 0$. In fact the latter congruence is a necessary and sufficient condition that the two forms $f, \varphi$ respectively be the following linear combinations of two squares in the field:

$$
f \equiv s \alpha_{x}^{2}+t \beta_{x}^{2} ; \quad \varphi \equiv \sigma a_{x}^{2}+\tau b_{x}^{2} .
$$

\section{§6. The Clebsch-Gordan Expansion.}

Gordan's series* is a case of expansion (2). It may be of interest to give a sketch of the derivation of this series from the present point of view, for two reasons: first to show how the preceding general theory applies, and secondly because our methods give a very compact treatment of the details of the derivation, which is believed to be novel.

We employ as symbolical basis the formal identity

$$
a_{x} b_{y}-a_{y} b_{x}=(a b)(x y) .
$$

From it we have

or

$$
a_{x}^{m} b_{y}^{n} \equiv\left[a_{y} b_{x}+(a b)(x y)\right]^{m} b_{y}^{n-m} \quad(n \geqq m),
$$

$$
\begin{aligned}
a_{x}^{m} b_{y}^{n}= & a_{y}^{m} b_{x}^{m} b_{y}^{n-m}+\left(\begin{array}{c}
m \\
1
\end{array}\right) a_{y}^{m-1} b_{x}^{m-1} b_{y}^{n-m}(a b)(x y)+\cdots \\
& +\left(\begin{array}{c}
m \\
k
\end{array}\right) a_{y}^{m-k} b_{x}^{m-k} b_{y}^{n-m}(a b)^{k}(x y)^{k}+\cdots+(a b)^{m}(x y)^{m} b_{y}^{n-m} .
\end{aligned}
$$

This is one form of Gordan's series. Here we have $(\S 1)$

$$
\Phi=a_{y}^{m} b_{x}^{m} b_{y}^{n-m}, \quad \nabla=\frac{\partial^{2}}{\partial x_{1} \partial y_{2}}-\frac{\partial^{2}}{\partial x_{2} \partial y_{1}} .
$$

But to reduce the series to the ordinary form we use the following polar formula:

$$
\text { (26) }=\frac{(a b)^{k}}{\left(\begin{array}{c}
m+n-2 k \\
n-k
\end{array}\right)} \sum_{k=0}^{m-k}\left(\begin{array}{c}
m-k \\
m-k-h
\end{array}\right)\left(\begin{array}{c}
n-k \\
n-m+h
\end{array}\right) a_{y}^{m-k-k} a_{x}^{k} b_{x}^{m-k-n} b_{y}^{n-m+k} \text {. }
$$

* Gordan, Ueber Combinanten, M a t h e ma t i s c h e A n nal en, vol. 5 (1872), p. 95. 
Since the sum of the numerical coefficients in the polar of a product is unity, (26) can be written

$$
\begin{aligned}
& \left(a_{x}^{m}, b_{x}^{n}\right)_{y-k}^{k}=(a b)^{k} a_{y}^{m-k} b_{x}^{n-k} b_{y}^{n-m} \\
& +\frac{(a b)^{k}}{\left(\begin{array}{c}
m+n-2 k \\
n-k
\end{array} \sum_{h=1}^{m-k}\left(\begin{array}{c}
m-k \\
m-k-h
\end{array}\right)\left(\begin{array}{c}
n-k \\
n-m+h
\end{array}\right) a_{y}^{m-k-h} b_{x}^{m-k-h} b_{y}^{n-m}\right.} \\
& \times\left(a_{x}^{h} b_{y}^{h}-a_{y}^{h} b_{x}^{h}\right) .
\end{aligned}
$$

The first term of this is, aside from the factor $\left(\begin{array}{c}m \\ k\end{array}\right)$, the coefficient of $(x y)^{k}$ in (25). Hence this coefficient is the $(n-k)$ th transvectant of $a_{x}^{m}$ and $b_{x}^{n}$, minus terms which contain the factor $(a b)^{k+1}(x y)$. Hence (27) may be used as a recursion formula; and, starting with $k=m$, we get

$$
\begin{aligned}
(a b)^{m} b_{y}^{n-m} & =\left(a_{x}^{m}, b_{x}^{n}\right)_{y^{n-m}}^{m}, \\
(a b)^{m-1} a_{y} b_{x} b_{y}^{n-m} & =\left(a_{x}^{m}, b_{x}^{n}\right)_{y^{n-m+1}}^{m-1}-\frac{1}{n-m+2}\left(a_{x}^{m}, b_{x}^{n}\right)_{y^{n-m}}^{m}(x y) \\
(a b)^{m-2} a_{y}^{2} b_{x}^{2} b_{y}^{n-m}= & \left(a_{x}^{m}, b_{x}^{n}\right)_{y^{n-m+2}}^{m-2}-\frac{4}{n-m+4}\left(a_{x}^{m}, b_{x}^{n}\right)_{y^{n-m+2}}^{m-1}(x y) \\
& +\frac{2}{(n-m+2)(n-m+3)}\left(a_{x}^{m}, b_{x}^{n}\right)_{y^{n-m}}^{m}(x y)^{2}
\end{aligned}
$$

And, in general, by induction

$$
\begin{aligned}
(a b)^{m-k} a_{y}^{k} b_{x}^{k} b_{y}^{n-m} & =\alpha_{0}\left(a_{x}^{m}, b_{x}^{n}\right)_{y^{m-m+k}}^{m-k}+\alpha_{1}\left(a_{x}^{m}, b_{x}^{n}\right)_{y^{n-m+k-1}}^{m-k+1}(x y)+\cdots \\
& +\alpha_{j}\left(a_{x}^{m}, b_{x}^{k}\right)_{y^{n-k+k+k}}^{m-k+j}(x y)^{j}+\cdots+\alpha_{k}\left(a_{x}^{m}, b_{x}^{n}\right)_{y^{n-m}}^{m}(x y)^{k}
\end{aligned}
$$

Consequently it follows at once from (25) that

$$
\begin{aligned}
a_{x}^{m} b_{y}^{n}=C_{0}( & \left.a_{x}^{m}, b_{x}^{n}\right)_{y^{n}}^{0}+C_{1}\left(a_{x}^{m}, b_{x}^{n}\right)_{y^{m-1}}^{1}(x y) \\
& +\cdots+C_{j}\left(a_{x}^{m}, b_{x}^{n}\right)_{y^{n-1}}^{j}(x y)^{j}+\cdots+C_{m}\left(a_{x}^{m}, b_{x}^{n}\right)_{y^{n-m}}^{m}(x y)^{m}
\end{aligned}
$$

The constants $C_{i}$ may now be determined in the usual manner by operating on both sides of (30) with the proper power of $\Delta$, and noting that when $y \sim x$ in any polar it goes back into the original polarized form. The following known formula* may be used for this purpose:

$$
\Delta^{i}(x y)^{j} \alpha_{x}^{n-j} \alpha_{y}^{m-j}=\frac{\lfloor j}{\underline{j-i}\lfloor\underline{\mid m+n-j-i+1}}(x y)^{j-i} \alpha_{x}^{n-j} \alpha_{y}^{m-j}
$$

* Grace and Young, Algebra of Invariants, p. 54. 
The coefficients may also be derived by multiplying the respective members of the column (28) by

$$
\left(\begin{array}{c}
m \\
m
\end{array}\right)(x y)^{m},\left(\begin{array}{c}
m \\
m-1
\end{array}\right)(x y)^{m-1}, \cdots,\left(\begin{array}{c}
m \\
2
\end{array}\right)(x y)^{2},\left(\begin{array}{c}
m \\
1
\end{array}\right)(x y), 1 ;
$$

and adding the results. Either method gives the well-known result.

$$
a_{x}^{m} b_{y}^{n}=\sum_{i=0}^{m} \frac{\left(\begin{array}{c}
m \\
i
\end{array}\right)\left(\begin{array}{c}
n \\
i
\end{array}\right)}{\left(\begin{array}{c}
m+n-i+1 \\
i
\end{array}\right)}(x y)^{i}\left(a_{x}^{m}, b_{x}^{n}\right)_{y^{n-1}}^{i} .
$$

The Univergity or Pennisgrutania,

Pemuadelpeia, Pa. 